\title{
A generalized Bachelier formula for pricing basket and spread options
}

\author{
Fulvia Fringuellotti ${ }^{1,2} \quad$ Ciprian Necula ${ }^{\ddagger, 2,3}$
}

November 16th, 2015

\begin{abstract}
In this paper we propose a closed-form pricing formula for European basket and spread options. Our approach is based on approximating the risk-neutral probability density function of the terminal value of the basket using a GaussHermite series expansion around the Gaussian density. The new method is quite general as it can be applied for a basket with a large number of assets and for all dynamics where the joint characteristic function of log-returns is known in closed form. We provide a simulation study to show the accuracy and the speed of our methodology.
\end{abstract}

Keywords: Basket options, Spread options, Option pricing, Gauss-Hermite series expansion

JEL Classification: C63, G13

Acknowledgements: We are grateful to Walter Farkas and Sebastian Matei for helpful comments. The research leading to these results has received funding from the European Union Seventh Framework Programme (FP7/2007-2013) under the ERC grant agreement 249415-RMAC and the MC-IEF grant agreement n. 627701.

\footnotetext{
${ }^{1}$ Swiss Finance Institute

${ }^{2}$ Department of Banking and Finance, University of Zurich, Plattenstrasse 14, CH-8032, Zurich, Switzerland

${ }^{3}$ Department of Money and Banking, DOFIN, Bucharest University of Economic Studies, Bucharest, Romania

${ }^{\ddagger}$ Corresponding author, email: ciprian.necula@bf.uzh.ch
} 


\section{Introduction}

Basket options are exotic options whose underlying is a basket composed by a weighted sum of different assets. Assuming that the basket is composed by $n$ assets, the payoff of a basket option is $\max \left\{\sum_{i=1}^{n} w_{i} S_{i}(T)-K, 0\right\}$, where $i \in\{1, \ldots, n\}$ is the subscript associated to each asset included in the basket, $w_{i}$ is the weight associated to the $i$-th underlying, $S_{i}(T)$ is the spot price of the $i$-th asset at the maturity date $T$ and $K$ is the strike price. These products are widely traded for hedging purposes. Indeed, they allow to hedge portfolio risk at a lower cost than a combined hedging strategy on each individual component of a portfolio. A popular subcategory of basket options are spread options, i.e. options whose basket is composed only by two assets and the weights are equal to 1 and -1 . The payoff of a spread option is given by $\max \left\{S_{1}(T)-S_{2}(T)-K, 0\right\}$. Spread options are extensively used in equity, fixed income, foreign exchange and commodity markets to hedge correlation risk among pairs of assets.

Pricing basket and spread options is quite challenging, as the distribution of the basket value is generally unknown even for parsimonious dynamics of the underlying assets. Consider, for instance, the simplest scenario that each of the underlying assets follows a log-normal process. Since a linear combination of log-normal processes is no longer log-normal, even in this trivial case, it is not possible to determine a closed-form pricing formula for the option price. The only case for which a closedform pricing formula actually exists is for exchange options (i.e. spread options with

strike price equal to zero) and it has been derived by Margrabe (1978). To circumvent this issue, academic research has focused the attention on two possible approaches to price the wide plethora of multi-asset options: numerical methods and analytic approximation models. 
The former include numerical integration methods, numerical solution to partial differential equations and Monte Carlo simulation. The best numerical approach we are aware of is the one by Hurd and Zhou (2010), who propose a numerical integration method for pricing European spread options in two or higher dimensions using the fast Fourier transform (FFT). On a similar track, Caldana and Fusai (2013) derive an approximate formula for European spread options, by extending the approach of Bjerksund and Stensland (2011) and using a univariate Fourier inversion. In general numerical methods provide more accurate prices. However, when the number of the underlying assets is relatively high, they become extremely slow and factually inapplicable. As a consequence, practitioners tend to prefer analytic approximation models, as they allow to get option prices quite quickly and they are suitable for risk management practices, as they permit to derive Greeks in a straightforward way.

Analytical approximation methods encompass a broad range of models that can be distinguished in two main categories: exercise boundary methods and moments matching models.

The former allow to retrieve the option price by approximating the non-linear exercise boundary. The popular approximation method by Kirk (1995) can be attributed to this class. More recent contributions by Carmona and Durrleman (2003), Deng et al. (2008), Li et al. (2012) and Bjerksund and Stensland (2011) provide a significant improvement in this direction.

Model matching models are based on the idea that the risk-neutral distribution of the underlying basket can be approximated by the method of moments. In this regard, Krekel et al. (2004) provide a comprehensive review of pricing models for European basket options proposed by Lévy (1992), Milevsky and Posner (1998a), Milevsky and Posner (1998b). Among newly proposed approximation methods, Borovkova et al. (2006) and Borovkova et al. (2007) come up with a valuation approach for European 
spread options and basket options that is based on the approximation of the basket distribution using the generalized family of log-normal distributions (GLN), whilst Deelstra et al. (2010) derive approximation formulae for Asian basket spread options using comonotonic bounds and moment matching techniques.

Further approximation methods are those of Gentle (1993), who approximates the basket payoff using a geometric average instead of an arithmetic average, of Beisser (1999), who derives the price of a basket call option as a weighted sum of artificial European call prices, of Venkatramanan and Alexander (2011) and Alexander and Venkatramanan (2012), who retrieve closed-form approximations for European spread options, basket options and rainbow options as the sum of the prices of compound exchange options. Closer to this work is the approximation method by Ju (2002), who proposes a pricing formula for European basket options based on a Taylor expansion of the ratio of the characteristic function of the arithmetic average to that of the approximating log-normal random variable around zero volatility.

The approach we propose in this paper is mainly based on approximating the risk-neutral density of the terminal value of the basket using an expansion around a density which is easy to compute. This methodology has been widely used for pricing options on a single asset. Jarrow and Rudd (1982) were the first to propose the density expansion approach using the Edgeworth series to expand the risk-neutral density of the terminal asset price around the log-normal density. Following a similar methodology, Corrado and $\mathrm{Su}(1996,1997)$ derived an option pricing formula using the Gram-Charlier Type A series to expand the risk-neutral density of the asset log-return around the Gaussian density. Starting with Corrado and Su (1996, 1997), the Gram-Charlier density expansion has become a popular tool to evaluate single asset options, as shown by Jurczenko, Maillet, and Negrea (2002). In spite of his straightforward implementation, the Gram-Charlier density expansion shows 
several drawbacks. Firstly, the probability density functions obtained according to the Gram-Charlier series expansion may yield negative probability values. This issue is not actually particularly severe, as Jondeau and Rockinger (2001) and Rompolis and Tzavalis (2007) have proposed two different methods to cope with it. A more relevant problem with the Gram-Charlier series expansion is that it lacks of convergence for heavy tailed distributions, which are those of major interest in finance. Indeed, Cramer (1957) showed that the Gram-Charlier Type A series expansion converges only if the speed of convergence of the probability density function is greater than $\exp \left(-x^{2} / 4\right)$ and this condition is satisfied only for a small class of distributions. This issue implies that, when approximating the density function of a fat-tailed distribution, the more terms are included in the Gram-Charlier series expansion, the worse will be the accuracy of the option price derived according to this approach. A possible way to tackle this problem is to consider a modified Gram-Chalier expansion, namely the Gauss-Hermite expansion, which is convergent for heavy tailed distribution as shown by Myller-Lebedeff (1907). The main difference between the Gram-Chalier expansion and the Gauss-Hermite expansion consists essentially in the adopted Hermite polynomial. Indeed, the Gram-Charlier Type A series expansions uses the "probabilits" Hermite polynomials while the Gauss-Hermite series expansion employs the "physicists" Hermite polynomials. Following the Gauss-Hermite expansion approach, Necula, Drimus, and Farkas (2013) derive a Black-Scholes-Merton-like formula for European options on a single asset by expanding the risk-neutral density of the log-returns of the underlying asset in a Gauss-Hermite series expansion. Recently the approximation of the risk-neutral density of the underlying has started to be used also in multi-assets option pricing. Paletta, Leccadito, and Tunaru (2013) derive a closed-form formula for pricing European basket options when assets follow a shifted log-normal process with jumps using a "probabilists" Hermite polynomial 
expansion of the probability density of the terminal value of the basket.

In this paper we extend the approach of Necula, Drimus, and Farkas (2013) to develop an approximation for pricing European basket and spread options. Assuming that the joint characteristic function of log-returns of the underlying assets is known in closed form, we derive a Bachelier-like option pricing formula using the Gauss-Hermite series expansion of the risk-neutral density of the terminal value of the basket around the Gaussian density.

The remainder of the paper is structured as follows. In section 2 we present the Gauss-Hermite series expansion. In section 3 we derive the Bachelier-like pricing formula for European call options and explain how it can be employed for pricing basket options. In section 4 we present a simulation study. The last section concludes. The appendix collects various proofs.

\section{The Gauss-Hermite series expansion}

The Gauss-Hermite series expansion allows to approximate the probability density function of a random variable by expanding it around the Gaussian density using "physicists" Hermite polynomials. Consider a random variable $X$ distributed according to a continuous and strictly increasing distribution function $F$ with mean $\mu$ and standard deviation $\sigma$. The probability density function of $X$ can be represented as a Gauss-Hermite series expansion around the Gaussian density as follows:

$$
f(x)=\frac{1}{\sigma} z\left(\frac{x-\mu}{\sigma}\right) \sum_{n=0}^{\infty} a_{n} H_{n}\left(\frac{x-\mu}{\sigma}\right)
$$

where $z(x)$ is the standard normal density, $a_{n}$ are the expansion coefficients and $H_{n}(x)$ denotes a $n$-th order "physicists" Hermite polynomial as defined in Abramowitz and Stegun (1964). The "physicists" Hermite polynomial can be obtained recursively by 
$H_{n+1}(x)=2 x H_{n}(x)-2 n H_{n-1}(x)$ with $H_{0}(x)=1$ and $H_{1}(x)=2 x$. It forms an orthogonal basis on $(-\infty,+\infty)$ with respect to the weight function $w(x)=e^{-x^{2}}$. Relying on this orthogonality condition, it is possible to show that the expansion coefficients are given by

$$
a_{n}=\frac{\sqrt{\pi}}{2^{n-1} n !} \int_{-\infty}^{\infty} z\left(\frac{x-\mu}{\sigma}\right) H_{n}\left(\frac{x-\mu}{\sigma}\right) f(x) d x
$$

and these coefficients result to be a linear combination of some weighted moments of $X$, namely $\mathbb{E}\left[\left(\frac{x-\mu}{\sigma}\right)^{n} z\left(\frac{x-\mu}{\sigma}\right)\right]$.

One issue with the Gauss-Hermite series expansion is that the integral of the approximated density function over the space where $X$ takes values is not necessarily equal to one. Indeed, this condition is satisfied only in the limit as it is equivalent to the identity $\sum_{k=0}^{\infty} a_{2 k} \frac{(2 k) !}{k !}=1$. Nevertheless, including a sufficiently high number of terms in the series expansion allows to prevent this problem. A possible alternative could be to normalize the expansion coefficients so that the truncated sum adds to one.

Similarly to the probability density function, also the characteristic function $\varphi$ of the random variable $X$, can be approximated using the Gauss-Hermite expansion with the same coefficients (e.g. Necula, Drimus, and Farkas, 2013):

$$
\varphi(\phi)=\exp \left(i \mu \phi-\frac{\sigma^{2}}{2} \phi^{2}\right) \sum_{n=0}^{\infty} a_{n} i^{n} H_{n}(\sigma \phi)
$$

where $i=\sqrt{-1}$. 


\section{A generalized Bachelier option pricing formula}

By expanding the risk-neutral density of the log-returns of the underlying asset in a Gauss-Hermite series expansion, Necula, Drimus, and Farkas (2013) derive a generalized Black-Scholes-Merton formula for European options. As the following result points out, the Gauss-Hermite series expansion of the terminal value of the underlying

allows to derive another type of closed form formula for pricing European options, a generalized Bachelier formula.

Proposition 1. (Generalized Bachelier option pricing formula) Assume that the riskneutral density of the terminal value of the underlying asset for the time horizon $\tau$ is characterized by mean $\mu:=\mu(t, \tau)$, standard deviation $\sigma:=\sigma(t, \tau)$ and GaussHermite expansion coefficients $\left(a_{n}:=a_{n}(t, \tau)\right)_{n \in \mathbb{N}}$.

Then the premium at time $t$ of a European call option with strike price $K$ and maturity $t+\tau$ is given by:

$$
c\left(\mu, \sigma, K, r, \tau ;\left\{a_{n}\right\}\right)=e^{-r \tau} \sum_{n=0}^{\infty} a_{n}\left\{(\mu-K) J_{n}+\sigma\left[H_{n}(-d) z(d)+2 n J_{n-1}\right]\right\}
$$

where $r$ is the risk-free interest rate, $d=(\mu-K) / \sigma$ and $J_{n}$ verifies the recursion relation $J_{n+1}=2 H_{n}(-d) z(d)+2 n J_{n-1}$ with $J_{0}=N(d)$ and $J_{1}=2 z(d)$ where $N($.$) is$ the cumulative distribution function of a standard normal variable.

Proof. see the Appendix.

Remark 1. Due to the martingale condition, one has that $\mu(t, \tau):=\mathbb{E}\left[S_{t+\tau}\right]=S_{t} e^{r \tau}$ where $S$ is the spot price on the underlying. Moreover, from equation (3), using the relation between the mean and the characteristic function, it follows that the GaussHermite coefficients have to observe the restriction $\sum_{n=1}^{\infty} n a_{n} i^{n} H_{n-1}(0)=0$. 
Remark 2. If the risk-neutral density of the terminal value is assumed Gaussian then the Gauss-Hermite expansion coefficients are given by $a_{0}=1$ and $a_{n}=0, n \geq 1$ and, therefore, equation (4) reduces to the Bachelier formula.

The generalized Bachelier formula (4) is particularly useful for pricing a European basket option when the joint characteristic function of the log-returns of the assets included in the basket is known in closed form. Since the value of the basket is given by a linear combination of asset prices, the moments of the terminal value of the underlying basket can be easily computed using the multinomial expansion formula. For exemplification, let us consider the case of a basket of two assets, $w_{1} S_{1}(t)+w_{2} S_{2}(t)$. Denote by $\varphi\left(\phi_{1}, \phi_{2}\right)$ the joint characteristic function of $\log$-prices, $\varphi\left(\phi_{1}, \phi_{2}\right):=\mathbb{E}\left[e^{\phi_{1} \ln \left(S_{1}(t+\tau)\right)+\phi_{2} \ln \left(S_{2}(t+\tau)\right)}\right]$, which is known in closed form. Using the binomial expansion formula, it follows that the $n$-th moment of the terminal value of the basket can be computed as $\mathbb{E}\left[\left(w_{1} S_{1}(t+\tau)+w_{2} S_{2}(t+\tau)\right)^{n}\right]=$ $\sum_{k=0}^{n}\left(\begin{array}{l}n \\ k\end{array}\right) w_{1}^{k} w_{2}^{n-k} \varphi(-i k,-i(n-k))$. The Gauss-Hermite expansion coefficients can be determined from the moments of the terminal value of the underlying basket using an intermediate procedure based on the type C Gram-Charlier series expansion (Muscolino and Ricciardi, 1999; Rompolis and Tzavalis, 2007). First, we compute the first 20 moments of the risk-neutral conditional distribution of the terminal value of the basket using the procedure described above. Next, we approximate the probability density function using the type C Gram-Charlier series expansion (C-GCSE) truncated after 10 terms * by computing the expansion coefficients of the C-GCSE from the first 20 moments using the method in Rompolis and Tzavalis (2007). In contrast with the classical type A Gram-Charlier expansion, the type C expansion guarantees that the values of the risk neutral density will be always positive. On the downside,

*A C-GCSE truncated after 10 terms provided good results in the simulation study. Increasing the number of terms in the truncation also increases the computational burden since a double number of moments are required to compute a given number of C-GCSE expansion coefficients. 
there is no closed form formula for option prices when approximating the risk neutral probability distribution function with a C-GCSE. Finally, the Gauss-Hermite coefficients can be computed from equation (2) using the C-GCSE approximation of the probability density function obtained in the previous step. A similar procedure was employed in Farkas, Necula, and Waelchli (2015) to obtain closed-form option prices in the context of a non-affine stochastic volatility model.

\section{Simulation study and preliminary results}

In this section we present a preliminary simulation analysis that gives support to the soundness of the proposed approach.

Let us consider the pricing of a European call option on the spread between two baskets (i.e. a basket spread option), where each basket is composed by three assets. The payoff of this option is given by $\max \left\{\widetilde{S}_{1}(T)-\widetilde{S}_{2}(T)-K, 0\right\}$ where $\widetilde{S}_{1}(T)=\sum_{i=1}^{3} w_{i} S_{i}(T)$ and $\widetilde{S}_{2}(T)=\sum_{i=4}^{6} w_{i} S_{i}(T)$. The dynamics of the assets is modeled by a multivariate Geometric Brownian Motion ${ }^{\dagger}$. The initial value of the six assets is equal to 100 u.m., so that the value of the spread at inception is zero. The time to maturity of the option is three months and the risk-free rate is set at $5 \%$. We consider three different values for the strike price, respectively $-5,0$ and 5 u.m. We take as the benchmark value of the basket spread option the price obtained through a Monte Carlo simulation with 10,000,000 paths. Figure 1 depicts the logarithm of the empirical density of the terminal value of the basket obtained through Monte Carlo simulations, along with the logarithm of the risk-neutral density approximated using the Gauss-Hermite series expansion truncated after 20 terms.

In Table 1 we report the benchmark prices obtained through Monte Carlo simulations and the option prices computed according to the proposed general Bachelier

\footnotetext{
${ }^{\dagger}$ The volatilities and the correlations were randomly generated.
} 
Figure 1: Logarithm of the empirical density of the terminal value of the basket obtained through Monte Carlo simulation and logarithm of the risk-neutral density approximated using the Gauss-Hermite series expansion around the Gaussian density.

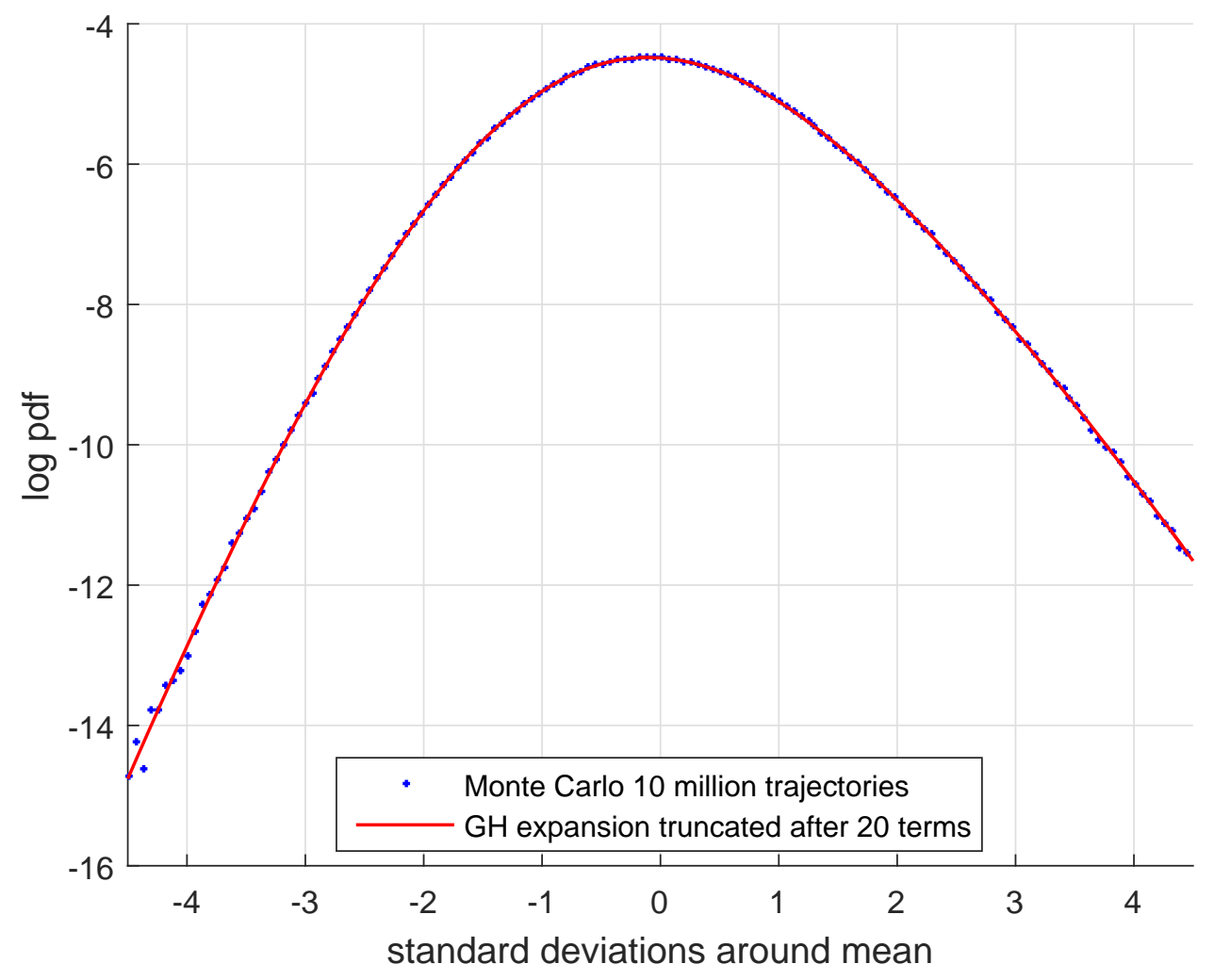


formula, along with the respective computation times ${ }^{\ddagger}$.

Table 1: Prices of call option on the spread between two baskets obtained according to Monte Carlo simulations (benchmark price) and on the basis of proposed closedform pricing formula. The value of the option is computed for three different strike prices. The number in brackets under each price obtained according to Monte Carlo simulations is the standard error. Computation time in seconds is displayed for each approach.

\begin{tabular}{|c|c|c|c|c|}
\hline \multirow{2}{*}{ Strike (u.m.) } & \multicolumn{2}{|c|}{ Monte Carlo } & \multicolumn{2}{c|}{ General Bachelier Formula } \\
\cline { 2 - 5 } & Price (u.m.) & Time (s) & Price (u.m.) & Time (s) \\
\hline-5 & $\begin{array}{r}16.7375 \\
(0.0076)\end{array}$ & 8.3386 & 16.7403 & 0.4211 \\
\hline 0 & 14.2115 & 8.1379 & 14.2062 & 0.4078 \\
$(0.0071)$ & & & \\
\hline 5 & 11.9546 & 8.2874 & 11.9498 & 0.4050 \\
\hline
\end{tabular}

The approximate value of the spread basket option obtained according to out pricing formula is considerably close to the benchmark value for all the strike prices taken into account. Beside the degree of accuracy, the proposed methodology shows a relevant gain in speed since the option price can be obtained roughly twenty time faster than using Monte Carlo simulations. This property is particularly convenient when it comes to price multi-assets options where the underlying basket is composed by many assets. Indeed, it would allow to overcome the computational issues related to numerical methods.

\footnotetext{
${ }^{\ddagger}$ Computation and programming have been done in MATLAB 2015b using a machine using Intel(R) Core(TM) i7-3632QM CPU 2.2GHz, 8GB RAM.
} 


\section{Concluding Remarks}

Basket options are exotic options whose underlying is a basket composed by a weighted sum of different assets. Pricing basket options is quite challenging, as the joint dynamics of the underlying assets is generally unknown. Academic research has focused the attention on two possible approaches to price basket options: numerical methods and analytic approximation models. In general, numerical methods provide more accurate prices, but when the number of the underlying assets is relatively high they become extremely slow and factually inapplicable. For this reason practitioners tend to prefer analytic approximation models. In this work we proposed a closed-form pricing formula to approximate the value of European basket and spread options. Our approach is based on using a Gauss-Hermite series expansion for the risk-neutral probability density function of the terminal value of the basket. The method can be applied for a basket with a large number of components and for all models where the joint characteristic function of log-returns is known in closed form. The simulation study pointed out the accuracy and the speed of the new methodology. 


\section{References}

Abramowitz, M. and I. A. Stegun (1964). Handbook of Mathematical Functions.

Alexander, C. and A. Venkatramanan (2012). Aoptions approximations for multiasset option pricing. Mathematical Finance 22(4), 667-689.

Beisser, J. (1999). Another way to value basket options.

Bjerksund, P. and G. Stensland (2011). Closed options spread option valuation. Quantitative Finance 14(10), 1-10.

Borovkova, S., F. J. Permana, and H. van der Weide (2006). Empirical analysis of analytic approximation approaches for pricing and hedging spread options. In M. Vanmaele, A. De Schepper, J. Dhaene, H. Reynaerts, W. Schoutens \& P. Van Goethem (E 4th Actuarial and Financial Mathematics Day 45-54. Wetteren, Belgie: Universa Press.

Borovkova, S., F. J. Permana, and H. van der Weide (2007). A closed form approach to the valuation and hedging of basket and spread options. The Journal of Derivatives 14(4), 8-24.

Caldana, R. and G. Fusai (2013). Agoption closed-form spread opton pricing formula. Journal of Banking and Finance 37, 4893-4906.

Carmona, R. and V. Durrleman (2003). Pricing and hedging spread options in a log-normal model.

Corrado, C. and T. Su (1996). S\&p 500 index option tests of jarrow and rudd's approximate option valuation formula. Journal of Futures Market 16, 611-629. 
Corrado, C. and T. Su (1997). Implied volatility skews and stock index skewness and kurtosis implied by s\&p 500 index option prices. Journal of Derivatives 4, 8-19.

Cramer, H. (1957). Mathematical Methods of Statistics.

Deelstra, G., A. Petkovic, and M. Vanmaele (2010). Pricing and hedging asian basket spread options. Journal of Computational and Applied Mathematics 233, 28142830.

Deng, S.-J., M. Li, and J. Zhou (2008). Closed-form appproximation for spread option prices and greeks.

Farkas, W., C. Necula, and B. Waelchli (2015). Herding and stochastic volatility. available at SSRN: http://dx.doi.org/10.2139/ssrn.2685939.

Gentle, D. (1993). Basket weaving. RISK 51-52.

Hurd, T. R. and Z. Zhou (2010). A fourier transform method for spread option pricing. SIAM Journal of Financial Mathematics 1, 142-15\%.

Jarrow, R. and A. Rudd (1982). Approximate option valuation for arbitrary stochastic processes. Journal of Financial Economics 10, 347-369.

Jondeau, E. and M. Rockinger (2001). Gram-charlier densities. Journal of Economic Dynamics and Control 25, 1457-1483.

Ju, E. (2002). Pricing asian and basket options via taylor expansion. Journal of Computational Finance 5(3), 79-103.

Jurczenko, E., B. Maillet, and B. Negrea (2002). Revisited multi-moment approximate option pricing models: A general comparison (discussion paper of the lse-fgm, n. $430)$. 
Kirk, E. (1995). Correlations in the energy markets. In: Managing energy price risk, Risk Publication and Enron.

Krekel, M., J. de Kock, and T.-K. Man (2004). An analysis of pricing methods for basket options. Wilmott magazine 82-89.

Lévy, E. (1992). Pricing european average rate currency options. Journal of International Money and Finance 11, 474-491.

Li, M., J. Zhou, and S.-J. Deng (2012). Multi-asset spread option pricing and hedging. Quantitative Finance 10(3), 305-324.

Margrabe, W. (1978). Toptions of an option to exchange one asset for another. Journal of Finance 33(1), 177-186.

Milevsky, M. A. and S. E. Posner (1998a). A closed-form approximation for valuing basket options. The Journal of Derivatives 54-61.

Milevsky, M. A. and S. E. Posner (1998b). Valuing exotic options by approximating the spd with higher moments. Journal of Financial Engineering 7(2), 54-61.

Muscolino, G. and G. Ricciardi (1999). Probability density function of mdof structural systems under non-normal delta-correlated inputs. Computer Methods in Applied Mechanics and Engineering 168, 121-133.

Myller-Lebedeff, W. (1907). Die theorie der integralgleichungen in anwendung auf einige reihnenentwicklungen. Mathematische Annalen 64, 388-416.

Necula, C., G. Drimus, and W. Farkas (2013). A general closed form option pricing formula. available at SSRN: http://dx.doi.org/10.2139/ssrn. 2210359. 
Paletta, T., A. Leccadito, and R. Tunaru (2013). Pricing and hedging basket options with exact moment matching. available at SSRN: http://papers.ssrn. com/sol3/papers.cfm?abstract_id=2368316.

Rompolis, E. S. and E. Tzavalis (2007). Retrieving risk neutral densities based on risk neutral moments through a gram-charlier series expansion. Mathematical and Computer Modelling 46, 225-234.

Venkatramanan, A. and C. Alexander (2011). Closed form approximations for spread options. Appied Mathematical Finance 18(5), 447-472. 


\section{Appendix}

\section{Proofs}

Proof of Proposition 1. If one denotes by $p_{t+\tau}\left(S_{t+\tau}\right)$ the terminal underlying price risk-neutral density and by $p(x)$ the corresponding standardized risk-neutral density then we have that:

$$
\begin{aligned}
c\left(\mu, \sigma, K, r, \tau ;\left\{a_{n}\right\}\right) & =e^{-r \tau} \int_{-\infty}^{\infty} \max \left(S_{t+\tau}-K, 0\right) p_{t+\tau}\left(S_{t+\tau}\right) d S_{t+\tau} \\
& =e^{-r \tau} \int_{-\infty}^{\infty} \max (\mu+\sigma x-K, 0) p(x) d x \\
& =e^{-r \tau} \int_{-d}^{\infty}(\mu+\sigma x-K) p(x) d x
\end{aligned}
$$

Taking into account the Gauss-Hermite expansion of the risk-neutral density, one has that $\int_{-d}^{\infty} p(x) d x=\sum_{n=0}^{\infty} a_{n} J_{n}$ with $J_{n}:=\int_{-d}^{\infty} H_{n}(x) z(x) d x$. At the same time $\int_{-d}^{\infty}(\mu+\sigma x) p(x) d x=\sum_{n=0}^{\infty} a_{n} \int_{-d}^{\infty}(\mu+\sigma x) H_{n}(x) z(x) d x$. Using integration by parts and the fact that $z^{\prime}(x)=-x z(x)$ it follows that $\int_{-d}^{\infty}(\mu+\sigma x) H_{n}(x) z(x) d x=\mu J_{n}+$ $\sigma\left[z(d) H_{n}(-d)+2 n J_{n-1}\right]$.

Using the properties of Hermite polynomials, namely $H_{n+1}(x)=2 x H_{n}(x)-2 n H_{n-1}(x)$, $H_{n}^{\prime}(x)=2 n H_{n-1}(x)$ and integration by parts, one can obtain the recursion equations for $J_{n}$ as follows:

$$
\begin{aligned}
J_{n+1} & =\int_{-d}^{\infty} H_{n+1}(x) z(x) d x=\int_{-d}^{\infty}\left(2 x H_{n}(x)-H_{n}^{\prime}(x)\right) z(x) d x \\
& =2 H_{n}(-d) z(d)+\int_{-d}^{\infty} H_{n}^{\prime}(x) z(x) d x \\
& =2 H_{n}(-d) z(d)+2 n \int_{-d}^{\infty} H_{n-1}(x) z(x) d x \\
& =2 H_{n}(-d) z(d)+2 n J_{n-1} .
\end{aligned}
$$


Finally, one has $J_{0}=\int_{-d}^{\infty} z(x) d x=N(d)$ and $J_{1}=\int_{-d}^{\infty} 2 x z(x) d x=2 z(d)$. 